\title{
Interval Point in Time Integer Data Type
}

National Cancer Institute

\section{Source}

National Cancer Institute. Interval Point in Time Integer Data Type. NCI Thesaurus. Code C95693.

A data type comprised of a set of consecutive values of an ordered point in time integer datatype. 